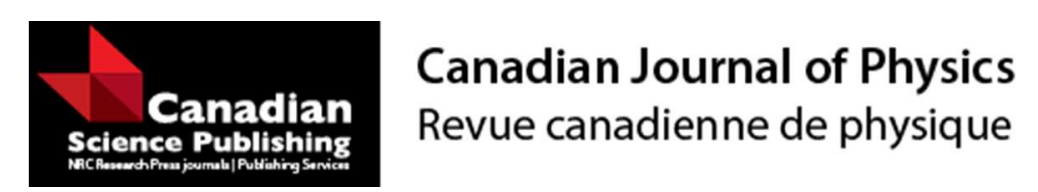

\title{
Spinor Field with Polynomial Nonlinearity in LRS Bianchi type-I spacetime
}

\begin{tabular}{|r|l|}
\hline Journal: & Canadian Journal of Physics \\
\hline Manuscript ID & cjp-2015-0574.R1 \\
\hline Manuscript Type: & Article \\
\hline Date Submitted by the Author: & 02-Oct-2015 \\
\hline Complete List of Authors: & $\begin{array}{l}\text { Saha, Bijan; Joint Institute for Nuclear Research, Laboratory of Information } \\
\text { technologies }\end{array}$ \\
\hline Keyword: & $\begin{array}{l}\text { Spinor field, dark energy, anisotropic cosmological models, isotropization, } \\
\text { polynomial nonlinearity }\end{array}$ \\
\hline \multicolumn{2}{|l}{} \\
\hline
\end{tabular}

SCHOLARONE ${ }^{\text {m }}$

Manuscripts 


\title{
Spinor Field with Polynomial Nonlinearity in LRS Bianchi type-I spacetime
}

\author{
Bijan Saha \\ Laboratory of Information Technologies \\ Joint Institute for Nuclear Research \\ 141980 Dubna, Moscow region, Russia*
}

\begin{abstract}
Within the scope of LRS Bianchi type-I cosmological model the role of spinor field on the evolution of the Universe is investigated. In doing so, we have considered a polynomial type of nonlinearity. It is found that depending on the sign of self-coupling constant the model allows either accelerated mode of expansion or oscillatory mode of evolution. While the non-diagonal components of the energy-momentum tensor of the spinor field in case of a full Bianchi type-I model leading to the vanishing mass and nonlinear term in the spinor field Lagrangian, in case of an LRS Bianchi type-I model neither mass term nor the nonlinear term of spinor field vanish.
\end{abstract}

PACS numbers: $98.80 . \mathrm{Cq}$

Keywords: Spinor field, dark energy, anisotropic cosmological models, isotropization, polynomial nonlinearity

\footnotetext{
*bijan@jinr.ru; http://spinor.bijansaha.ru
} 
Saha B.

\section{INTRODUCTION}

Recently, after some remarkable works by different authors [1-15], showing the important role that spinor fields play on the evolution of the Universe, the situation began to change. This change of attitude is directly related to some fundamental questions of modern cosmology: (i) problem of initial singularity; (ii) problem of isotropization and (iii) late time acceleration of the Universe.

Given the role that spinor field can play in the evolution of the Universe, question that naturally pops up is, if the spinor field can redraw the picture of evolution caused by perfect fluid and dark energy, is it possible to simulate perfect fluid and dark energy by means of a spinor field? Affirmative answer to this question was given in the a number of papers [16-20]. In those papers spinor description of matter such as perfect fluid and dark energy was given and the evolution of the Universe given by different Bianchi models was thoroughly studied. In almost all the papers the spinor field was considered to be time-dependent functions and its energy-momentum tensor was given by the diagonal elements only.

Some latest study shows that due to the specific connection with gravitational field the energymomentum tensor of the spinor field possesses non-trivial non-diagonal components as well and these non-zero non-diagonal components of energy-momentum tensor play decisive role on the character of geometry of spacetime as well as on the components of spinor field [21-24].

In this paper we study the evolution of the Universe filled with spinor field within the scope of a LRS Bianchi type-I cosmological model. It should be noted that a general Bianchi type-I model in presence of a nonlinear spinor field dully evolves into a LRS Bianchi type-I model [22]. In this paper we thoroughly study in role of spinor field in the evolution of the Universe given by a LRS Bianchi type-I model. Here we also consider a more general type of nonlinearity.

\section{BASIC EQUATIONS}

In this report we plan to study the evolution of the Universe given by a Locally Rotationally Symmetric Bianchi type-I anisotropic cosmological model filled with nonlinear spinor field.

The LRS Bianchi type-I (BI) model is the ordinary Bianchi type-I model with two of the three metric functions being equal to each other and can be given by

$$
d s^{2}=d t^{2}-a_{1}^{2}\left[d x^{2}+d y^{2}\right]-a_{3}^{2} d z^{2},
$$

with $a_{1}$ and $a_{3}$ being the functions of time only. 
The nontrivial components of the Einstein tensor corresponding to the metric (2.1) are

$$
\begin{aligned}
G_{1}^{1} & =G_{2}^{2}=-\left(\frac{\ddot{a}_{3}}{a_{3}}+\frac{\ddot{a}_{1}}{a_{1}}+\frac{\dot{a}_{3}}{a_{3}} \frac{\dot{a}_{1}}{a_{1}}\right), \\
G_{3}^{3} & =-\left(2 \frac{\ddot{a}_{1}}{a_{1}}+\frac{\dot{a}_{1}^{2}}{a_{1}^{2}}\right), \\
G_{0}^{0} & =-\left(\frac{\dot{a}_{1}^{2}}{a_{1}^{2}}+2 \frac{\dot{a}_{3}}{a_{3}} \frac{\dot{a}_{1}}{a_{1}}\right) .
\end{aligned}
$$

Keeping this in mind the symmetry between $\psi$ and $\bar{\psi}$ we choose the symmetrized Lagrangian [25] for the spinor field as [5]:

$$
L=\frac{l}{2}\left[\bar{\psi} \gamma^{\mu} \nabla_{\mu} \psi-\nabla_{\mu} \bar{\psi} \gamma^{\mu} \psi\right]-m_{\mathrm{sp}} \bar{\psi} \psi-F,
$$

where the nonlinear term $F$ describes the self-interaction of a spinor field and can be presented as some arbitrary functions of invariants generated from the real bilinear forms of a spinor field. We consider $F=F(K)$, with $K$ taking one of the following expressions $\{I, J, I+J, I-J\}$. It can be shown that such a choice describes the nonlinearity in its most general form.

Here $\nabla_{\mu}$ is the covariant derivative of spinor field:

$$
\nabla_{\mu} \psi=\frac{\partial \psi}{\partial x^{\mu}}-\Gamma_{\mu} \psi, \quad \nabla_{\mu} \bar{\psi}=\frac{\partial \bar{\psi}}{\partial x^{\mu}}+\bar{\psi} \Gamma_{\mu}
$$

with $\Gamma_{\mu}$ being the spinor affine connection. In (2.3) $\gamma^{\prime}$ s are the Dirac matrices in curve space-time and obey the following algebra

$$
\gamma^{\mu} \gamma^{v}+\gamma^{v} \gamma^{\mu}=2 g^{\mu v}
$$

and are connected with the flat space-time Dirac matrices $\bar{\gamma}$ in the following way

$$
g_{\mu v}(x)=e_{\mu}^{a}(x) e_{\nu}^{b}(x) \eta_{a b}, \quad \gamma_{\mu}(x)=e_{\mu}^{a}(x) \bar{\gamma}_{a}
$$

where $\eta_{a b}=\operatorname{diag}(1,-1,-1,-1)$ and $e_{\mu}^{a}$ is a set of tetrad 4-vectors. The spinor affine connection matrices $\Gamma_{\mu}(x)$ are uniquely determined up to an additive multiple of the unit matrix by the equation

$$
\nabla_{\mu} \gamma_{v}=\frac{\partial \gamma_{v}}{\partial x^{\mu}}-\Gamma_{v \mu}^{\rho} \gamma_{\rho}-\Gamma_{\mu} \gamma_{v}+\gamma_{v} \Gamma_{\mu}=0
$$

with the solution

$$
\Gamma_{\mu}=\frac{1}{4} \bar{\gamma}_{a} \gamma^{v} \partial_{\mu} e_{v}^{(a)}-\frac{1}{4} \gamma_{\rho} \gamma^{v} \Gamma_{\mu \nu}^{\rho}
$$


The spin affine connection corresponding to LRS BI metric (2.1) can be written explicitly as

$$
\Gamma_{0}=0, \quad \Gamma_{1}=\frac{\dot{a}_{1}}{a_{1}} \bar{\gamma}^{1} \bar{\gamma}^{0}, \quad \Gamma_{2}=\frac{\dot{a}_{1}}{a_{1}} \bar{\gamma}^{2} \bar{\gamma}^{0}, \quad \Gamma_{3}=\frac{\dot{a}_{3}}{a_{3}} \bar{\gamma}^{3} \bar{\gamma}^{0}
$$

Varying (2.3) with respect to $\bar{\psi}(\psi)$ one finds the spinor field equations:

$$
\begin{aligned}
& \imath \gamma^{\mu} \nabla_{\mu} \psi-m_{\mathrm{sp}} \psi-2 F_{K}\left(S K_{I}+\imath P K_{J} \gamma^{5}\right) \psi=0 \\
& \imath \nabla_{\mu} \bar{\psi} \gamma^{\mu}+m_{\mathrm{sp}} \bar{\psi}+2 F_{K} \bar{\psi}\left(S K_{I}+\imath P K_{J} \gamma^{5}\right)=0 .
\end{aligned}
$$

Here we denote $F_{K}=d F / d K, K_{I}=d K / d I$ and $K_{J}=d K / d J$.

The energy-momentum tensor of the spinor field is given by

$$
T_{\mu}^{\rho}=\frac{i}{4} g^{\rho \nu}\left(\bar{\psi} \gamma_{\mu} \nabla_{\nu} \psi+\bar{\psi} \gamma_{\nu} \nabla_{\mu} \psi-\nabla_{\mu} \bar{\psi} \gamma_{\nu} \psi-\nabla_{\nu} \bar{\psi} \gamma_{\mu} \psi\right)-\delta_{\mu}^{\rho} L_{\mathrm{sp}}
$$

where $L_{\mathrm{sp}}$ in view of (2.10a) and (2.10b) can be rewritten as

$$
\begin{aligned}
L_{\mathrm{sp}} & =\frac{l}{2}\left[\bar{\psi} \gamma^{\mu} \nabla_{\mu} \psi-\nabla_{\mu} \bar{\psi} \gamma^{\mu} \psi\right]-m_{\mathrm{sp}} \bar{\psi} \psi-F(K) \\
& =\frac{l}{2} \bar{\psi}\left[\gamma^{\mu} \nabla_{\mu} \psi-m_{\mathrm{sp}} \psi\right]-\frac{l}{2}\left[\nabla_{\mu} \bar{\psi} \gamma^{\mu}+m_{\mathrm{sp}} \bar{\psi}\right] \psi-F(K), \\
& =2 F_{K}\left(I K_{I}+J K_{J}\right)-F=2 K F_{K}-F(K) .
\end{aligned}
$$

Further inserting (2.4) into (2.11) the energy-momentum tensor of the spinor field can be written as

$$
\begin{aligned}
T_{\mu}{ }^{\rho} & =\frac{l}{4} g^{\rho v}\left(\bar{\psi} \gamma_{\mu} \partial_{v} \psi+\bar{\psi} \gamma_{\nu} \partial_{\mu} \psi-\partial_{\mu} \bar{\psi} \gamma_{v} \psi-\partial_{\nu} \bar{\psi} \gamma_{\mu} \psi\right) \\
& -\frac{l}{4} g^{\rho v} \bar{\psi}\left(\gamma_{\mu} \Gamma_{v}+\Gamma_{v} \gamma_{\mu}+\gamma_{v} \Gamma_{\mu}+\Gamma_{\mu} \gamma_{v}\right) \psi-\delta_{\mu}^{\rho}\left(2 K F_{K}-F(K)\right)
\end{aligned}
$$

Finally, exploiting the explicit form of spin connection (2.9) after some manipulations one find the following non-trivial components of the energy-momentum tensor of the spinor field

$$
\begin{aligned}
& T_{0}^{0}=m_{\mathrm{sp}} S+F(K), \\
& T_{1}^{1}=T_{2}^{2}=T_{3}^{3}=F(K)-2 K F_{K}, \\
& T_{3}^{1}=\frac{l}{4} \frac{a_{3}}{a_{1}}\left(\frac{\dot{a}_{3}}{a_{3}}-\frac{\dot{a}_{1}}{a_{1}}\right) \bar{\psi} \bar{\gamma}^{3} \bar{\gamma}^{1} \bar{\gamma}^{0} \psi=\frac{1}{4} \frac{a_{3}}{a_{1}}\left(\frac{\dot{a}_{3}}{a_{3}}-\frac{\dot{a}_{1}}{a_{1}}\right) A^{2}, \\
& T_{3}^{2}=\frac{l}{4} \frac{a_{3}}{a_{1}}\left(\frac{\dot{a}_{1}}{a_{1}}-\frac{\dot{a}_{3}}{a_{3}}\right) \bar{\psi} \bar{\gamma}^{2} \bar{\gamma}^{3} \bar{\gamma}^{0} \psi=\frac{1}{4} \frac{a_{3}}{a_{1}}\left(\frac{\dot{a}_{1}}{a_{1}}-\frac{\dot{a}_{3}}{a_{3}}\right) A^{1} .
\end{aligned}
$$


So the complete set of Einstein equation for BI metric should be

$$
\begin{aligned}
\frac{\ddot{a}_{3}}{a_{3}}+\frac{\ddot{a}_{1}}{a_{1}}+\frac{\dot{a}_{3}}{a_{3}} \frac{\dot{a}_{1}}{a_{1}} & =\kappa\left(F(K)-2 K F_{K}\right), \\
2 \frac{\ddot{a}_{1}}{a_{1}}+\frac{\dot{a}_{1}^{2}}{a_{1}^{2}} & =\kappa\left(F(K)-2 K F_{K}\right), \\
\frac{\dot{a}_{1}^{2}}{a_{1}^{2}}+2 \frac{\dot{a}_{3}}{a_{3}} \frac{\dot{a}_{1}}{a_{1}} & =\kappa\left(m_{\mathrm{sp}} S+F(K)\right), \\
0 & =\left(\frac{\dot{a}_{3}}{a_{3}}-\frac{\dot{a}_{1}}{a_{1}}\right) A^{2}, \\
0 & =\left(\frac{\dot{a}_{1}}{a_{1}}-\frac{\dot{a}_{3}}{a_{3}}\right) A^{1} .
\end{aligned}
$$

Before solving the Einstein equations let us first write the equations for the bilinear spinor forms. Recalling there are 16 bilinear spinor forms, namely $S=\bar{\psi} \psi, \quad P=\imath \bar{\psi} \gamma^{5} \psi, \quad v^{\mu}=$ $\bar{\psi} \gamma^{\mu} \psi, \quad A^{\mu}=\bar{\psi} \gamma^{5} \gamma^{\mu} \psi$ and $Q^{\mu v}=\bar{\psi} \sigma^{\mu v} \psi$ are the scalar, pseudoscalar, vector, pseudovector and antisymmetric tensor, respectively, for the LRS BI metric one finds the following system of equations:

$$
\begin{aligned}
\dot{S}_{0}+\mathscr{G} A_{0}^{0} & =0, \\
\dot{P}_{0}-\Phi A_{0}^{0} & =0, \\
\dot{A}_{0}^{0}+\Phi P_{0}-\mathscr{G} S_{0} & =0, \\
\dot{A}_{0}^{3} & =0, \\
\dot{v}_{0}^{0} & =0, \\
\dot{v}_{0}^{3}+\Phi Q_{0}^{30}+\mathscr{G} Q_{0}^{21} & =0, \\
\dot{Q}_{0}^{30}-\Phi v_{0}^{3} & =0, \\
\dot{Q}_{0}^{21}-\mathscr{G} v_{0}^{3} & =0,
\end{aligned}
$$

where we denote $S_{0}=S V, P_{0}=P V, A_{0}^{\mu}=A_{\mu} V, v_{0}^{\mu}=v^{\mu} V, Q_{0}^{\mu v}=Q^{\mu v} V$ and $\Phi=m_{\mathrm{sp}}+\mathscr{D}$. We also denote $\mathscr{D}=2 S F_{K} K_{I}$ and $\mathscr{G}=2 P F_{K} K_{J}$, with $F_{K}=d F / d K, K_{I}=d K / d I$ and $K_{J}=d K / d J$.

Here we also introduce the volume scale

$$
V=a_{1}^{2} a_{3}
$$




\section{SOLUTION TO THE FIELD EQUATIONS}

From $(2.16 \mathrm{a})-(2.16 \mathrm{~h})$ one finds the following relations:

$$
\begin{aligned}
\left(S_{0}\right)^{2}+\left(P_{0}\right)^{2}+\left(A_{0}^{0}\right)^{2} & =C_{1}=\text { Const }, \\
A_{0}^{3} & =C_{2}=\text { Const }, \\
v_{0}^{0} & =C_{3}=\text { Const }, \\
\left(Q_{0}^{30}\right)^{2}+\left(Q_{0}^{21}\right)^{2}+\left(v_{0}^{3}\right)^{2} & =C_{4}=\text { Const. }
\end{aligned}
$$

Let us now go back to Einstein equations. The off-diagonal components of the Einstein equations (2.15d) and (2.15e) impose the following restrictions either on the components of the spinor field or on the metric functions:

$$
\begin{aligned}
A^{2}=0, \quad A^{1} & =0, \\
\frac{\dot{a}_{3}}{a_{3}}-\frac{\dot{a}_{1}}{a_{1}} & =0 .
\end{aligned}
$$

The restriction (3.2b) leads to $a_{3}=q_{0} a_{1}$ with $q_{0}$ being some constant. In this case the system can be described by a FRW model from the very beginning. Here we don't consider this case. WE will do it later within the scope of a FRW model.

We consider the case when the restriction is imposed on the components of the spinor field in details. Subtraction of (2.15b) from (2.15a) gives

$$
\frac{\ddot{a}_{3}}{a_{3}}-\frac{\ddot{a}_{1}}{a_{1}}+\frac{\dot{a}_{1}}{a_{1}}\left(\frac{\dot{a}_{3}}{a_{3}}-\frac{\dot{a}_{1}}{a_{1}}\right)=0
$$

that leads to [5]

$$
a_{1}=D_{1} V^{1 / 3} \exp \left(X_{1} \int \frac{d t}{V}\right), \quad a_{3}=\left(1 / D_{1}^{2}\right) V^{1 / 3} \exp \left(-2 X_{1} \int \frac{d t}{V}\right) .
$$

with $D_{1}$ and $X_{1}$ being the integration constants. Thus we see that the metric functions can be expressed in terms of $V$.

The solutions to the spinor field equations (2.10a) in this case can be presented as [5]

$$
\psi_{1,2}(t)=\frac{C_{1,2}}{\sqrt{V}} \exp \left(-i \int \Phi d t\right), \quad \psi_{3,4}(t)=\frac{C_{3,4}}{\sqrt{V}} \exp \left(i \int \Phi d t\right),
$$

with $C_{1}, C_{2}, C_{3}, C_{4}$ being the integration constants and related to $V_{0}$ as

$$
C_{1}^{*} C_{1}+C_{2}^{*} C_{2}-C_{3}^{*} C_{3}-C_{4}^{*} C_{4}=V_{0}
$$


Here we have assumed that $K=I$, i.e., $F=F(I)$. The reason for this choice is discussed later.

Thus we see that the metric functions, the components of spinor field as well as the invariants constructed from metric functions and spinor fields are some inverse functions of $V$ of some degree. Hence at any spacetime point where $V=0$ it is a singular point. So it is important to study the behavior of $V$ which we do in the next section.

\section{RESULTS AND DISCUSSIONS}

In this section we discus the results obtained in the previous section. In doing so we pay special attention to the volume scale $V$.

Let us first see, whether the model becomes asymptotically isotropic. It can be shown that for an expanding Universe, when $V \rightarrow \infty$ as $t \rightarrow \infty$, the isotropization process of the Universe takes place. To prove that we exploit the isotropization condition proposed in [26]

$$
\left.\frac{a_{i}}{a}\right|_{t \rightarrow \infty} \rightarrow \text { const. }
$$

Then by rescaling some of the coordinates, we can make $a_{i} / a \rightarrow 1$, and the metric will become manifestly isotropic at large $t$.

Taking into account that $a=V^{1 / 3}$ from (3.4) we find

$$
\frac{a_{1}}{a}=D_{1} \exp \left(X_{1} \int \frac{d t}{V}\right) \rightarrow D_{1}, \quad \frac{a_{3}}{a}=\left(1 / D_{1}^{2}\right) \exp \left(-2 X_{1} \int \frac{d t}{V}\right) \rightarrow 1 / D_{1}^{2},
$$

as $V \rightarrow \infty$. Recall that the isotropic FRW model has same scale factor in all three directions, i.e., $a_{1}(t)=a_{2}(t)=a_{3}(t)=a(t)$. So for the LRS BI universe to evolve into a FRW one we should have $D_{1}=1$. Moreover, the isotropic nature of the present Universe leads to the fact that $\left|X_{1}\right|<<1$, so that $\int[V(t)]^{-1} d t \rightarrow 0$ for $t<\infty$ (for $V(t)=t^{n}$ with $n>1$ the integral tends to zero as $t \rightarrow \infty$ ).

Our next step will be to define $V$. Combining the diagonal Einstein equations (2.15a), (2.15b) and $(2.15 \mathrm{c})$ in a certain way for $V$ we find [5]

$$
\ddot{V}=\frac{3 \kappa}{2}\left(m_{\mathrm{sp}} S+2\left(F(K)-K F_{K}\right)\right) V .
$$

Now in order to solve (4.3) we have to know the relation between the spinor and the gravitational fields. Using the equations (2.16a) and (2.16b) it can be show that

$$
K=\frac{V_{0}^{2}}{V^{2}}
$$


The relation (4.4) holds only for massless spinor field if $K$ takes one of the following expressions $\{J, I+J, I-J\}$, while for $K=I$ it holds both for massless and massive spinor field. In case of $K=I+J$ one can write $S=\sin \left(V_{0} / V\right)$ and $P=\cos \left(V_{0} / V\right)$, whereas for $K=I-J$ one can write $S=\cosh \left(V_{0} / V\right)$ and $P=\sinh \left(V_{0} / V\right)$. In what follows, we will consider the case for $K=I$, as in this case further setting spinor mass $m_{\mathrm{sp}}=0$ we can revive the results for other cases. Assuming

$$
F=\sum_{k} \lambda_{k} I^{n_{k}}=\sum_{k} \lambda_{k} S^{2 n_{k}}
$$

on account of $S=V_{0} / V$ we find

$$
\ddot{V}=\frac{3 \kappa}{2}\left[m_{\mathrm{sp}} V_{0}+2 \sum_{k} \lambda_{k}\left(1-n_{k}\right) V_{0}^{2 n_{k}} V^{1-2 n_{k}}\right],
$$

with the solution in quadrature

$$
\int \frac{d V}{\sqrt{3 \kappa\left[m_{\mathrm{sp}} V_{0} V+\sum_{k} \lambda_{k} V_{0}^{2 n_{k}} V^{2\left(1-n_{k}\right)}\right]+\bar{C}}}=t+t_{0}
$$

with $\bar{C}$ and $t_{0}$ being some arbitrary constants.

Thus we see that the metric functions, the components of spinor field as well as the invariants constructed from metric functions and spinor fields are some inverse functions of $V$ of some degree. Hence at any spacetime point where $V=0$ it is a singular point. So we consider the initial value of $V(0)$ is small but non-zero. As a result for the nonlinear term to prevail in (4.6) we should have $n_{k}=n_{1}: 1-2 n_{1}<0$, i.e., $n_{1}>1 / 2$, whereas for an expanding Universe when $V \rightarrow \infty$ as $t \rightarrow \infty$ one should have $n_{k}=n_{2}: 1-2 n_{2}>0$, i.e., $n_{2}<1 / 2$. As is seen from (4.6), $n_{k}=n_{0}: n_{0}=1 / 2$ leads to a term that can be added to the mass term.

In this case we obtain

$$
\begin{aligned}
\ddot{V} & =\Phi_{1}(V), \\
\Phi_{1}(V) & =\frac{3 \kappa}{2}\left[\left(m_{\mathrm{sp}}+\lambda_{0}\right) V_{0}+2 \lambda_{1}\left(1-n_{1}\right) V_{0}^{2 n_{1}} V^{1-2 n_{1}}+2 \lambda_{2}\left(1-n_{2}\right) V_{0}^{2 n_{2}} V^{1-2 n_{2}}\right] .
\end{aligned}
$$

Equation (4.8) allows the first integral

$$
\begin{aligned}
\dot{V} & =\Phi_{2}(V), \\
\Phi_{2}(V) & =\sqrt{3 \kappa\left[\left(m_{\mathrm{sp}}+\lambda_{0}\right) V_{0} V+\lambda_{1} V_{0}^{2 n_{1}} V^{2\left(1-n_{1}\right)}+\lambda_{2} V_{0}^{2 n_{2}} V^{2\left(1-n_{2}\right)}+\bar{C}\right]} .
\end{aligned}
$$


The solution to the equation (4.8) can be written in quadrature as follows

$$
\int \frac{d V}{\Phi_{2}(V)}=t+t_{0}
$$

To solve the (4.8) we should choose the problem parameters $V_{0}, m_{\mathrm{sp}}, \kappa, \bar{C}, \lambda_{k}$ as well as the initial value of $V(0)$ in such as way that does not leads to

$$
\left(m_{\mathrm{sp}}+\lambda_{0}\right) V_{0} V+\lambda_{1} V_{0}^{2 n_{1}} V^{2\left(1-n_{1}\right)}+\lambda_{2} V_{0}^{2 n_{2}} V^{2\left(1-n_{2}\right)}+\bar{C}<0 .
$$

For simplicity let us set $V_{0}=1, m_{\mathrm{sp}}=1, C_{0}=10, \kappa=1$. In line with out discussion above we consider $n_{0}=1 / 2, n_{1}=2$ and $n_{2}=0$. In our case we set $V(0)=0.5$. We set $\lambda_{0}=1$, whereas $\lambda_{1}= \pm 1$ and $\lambda_{2}= \pm 1$ were taken in different combinations. It was found that depending of the sign of $\lambda_{2}$ the model gives principally different type of solutions, namely, in case of positive $\lambda_{2}$ we have an accelerated mode of expansion of the Universe, while for negative $\lambda_{2}$ we have oscillatory solution.

Defining deceleration parameter

$$
q=-\frac{V \ddot{V}}{\dot{V}^{2}}=-\frac{V \Phi_{1}(V)}{\Phi_{2}^{2}(V)},
$$

from (4.8) and (4.9) we have

$$
q=-\frac{\frac{3 \kappa}{2}\left[\left(m_{\mathrm{sp}}+\lambda_{0}\right) V_{0} V+2 \lambda_{1}\left(1-n_{1}\right) V_{0}^{2 n_{1}} V^{2\left(1-n_{1}\right)}+2 \lambda_{2}\left(1-n_{2}\right) V_{0}^{2 n_{2}} V^{2\left(1-n_{2}\right)}\right]}{3 \kappa\left[\left(m_{\mathrm{sp}}+\lambda_{0}\right) V_{0} V+\lambda_{1} V_{0}^{2 n_{1}} V^{2\left(1-n_{1}\right)}+\lambda_{2} V_{0}^{2 n_{2}} V^{2\left(1-n_{2}\right)}+\bar{C}\right]} .
$$

Taking into account that for an expanding Universe at large $t$ the term $V^{1-2 n_{2}}$ prevails, for deceleration parameter we find

$$
\lim _{V \rightarrow \infty} q \longrightarrow-\left(1-n_{2}\right)<0, \quad \text { since } \quad n_{2}<1 / 2 \text {. }
$$

Thus we see that spinor field nonlinearity generates late time acceleration of the Universe.

In Fig. 1 and Fig. 2 we have plotted the evolution of volume scale $V$ for a positive and negative self-coupling constant $\lambda_{2}$, respectively. As one sees from Fig. 1, a positive $\lambda_{2}$ gives rise to a accelerated mode of expansion, whereas Fig. 2 with negative $\lambda_{2}$ shows the oscillatory mode of expansion. In Fig. 3 the picture of deceleration parameter $q$ are illustrated for a positive $\lambda_{2}$.

Finally we study what happens to shear and anisotropic parameter in this case. In doing so, let us first rewrite the corresponding quantities. The expansion $\vartheta$ for LRS BI metric reads

$$
\vartheta=2 \frac{\dot{a}_{1}}{a_{1}}+\frac{\dot{a}_{3}}{a_{3}}=\frac{\dot{V}}{V},
$$




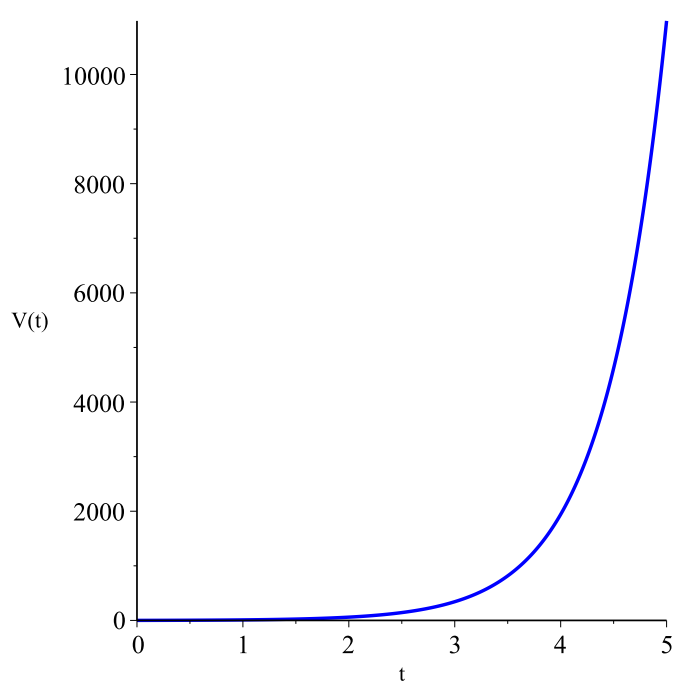

FIG. 1. Evolution of the Universe for a positive $\lambda_{2}$

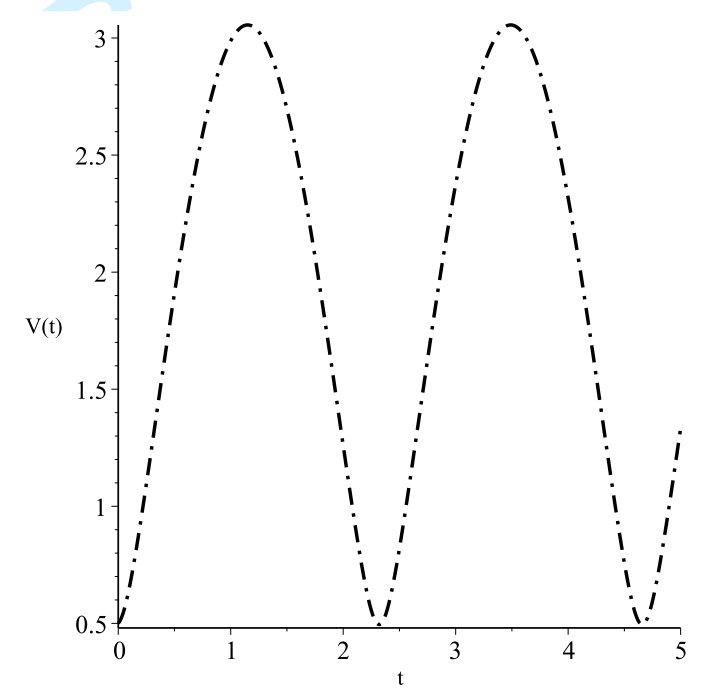

FIG. 2. Evolution of the Universe for a negative $\lambda_{2}$

whereas, from

$$
\begin{aligned}
& \sigma_{1}^{1}=\sigma_{2}^{2}=\frac{\dot{a}_{1}}{a_{1}}-\frac{1}{3} \vartheta=\frac{1}{3}\left(\frac{\dot{a}_{1}}{a_{1}}-\frac{\dot{a}_{3}}{a_{3}}\right), \\
& \sigma_{3}^{3}=\frac{\dot{a}_{3}}{a_{3}}-\frac{1}{3} \vartheta=-\frac{2}{3}\left(\frac{\dot{a}_{1}}{a_{1}}-\frac{\dot{a}_{3}}{a_{3}}\right)
\end{aligned}
$$

one finds the expression for shear

$$
\sigma^{2}=\frac{1}{2}\left[\sum_{i=1}^{3}\left(\frac{\dot{a}_{i}}{a_{i}}\right)^{2}-\frac{1}{3} \vartheta^{2}\right]=\frac{1}{3}\left(\frac{\dot{a}_{1}}{a_{1}}-\frac{\dot{a}_{3}}{a_{3}}\right)^{2} .
$$




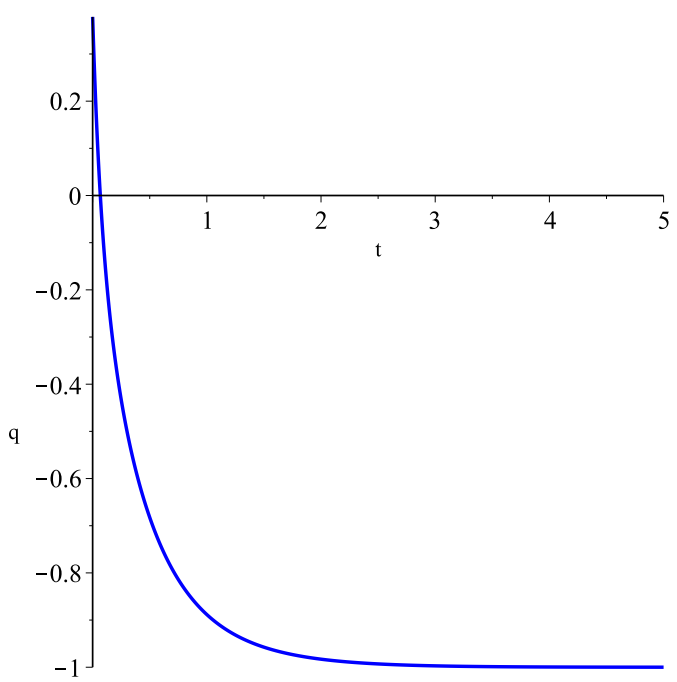

FIG. 3. Plot of deceleration parameter $q$ for a positive $\lambda_{2}$

The anisotropic parameter in this case has the form

$$
A_{m}=\frac{1}{3} \sum_{i=1}^{3}\left(\frac{H_{i}}{H}-1\right)^{2}=\frac{1}{3 H^{2}}\left[2\left(\frac{\dot{a}_{1}}{a_{1}}\right)^{2}+\left(\frac{\dot{a}_{3}}{a_{3}}\right)^{2}\right]-1
$$

where $H=\frac{1}{3}\left(2 \frac{\dot{a}_{1}}{a_{1}}+\frac{\dot{a}_{3}}{a_{3}}\right)=\frac{1}{3} \frac{\dot{V}}{V}$. Further from (3.4) we find that

$$
\frac{\dot{a}_{1}}{a_{1}}=\frac{1}{3} \frac{\dot{V}}{V}+\frac{X_{1}}{V}, \quad \frac{\dot{a}_{3}}{a_{3}}=\frac{1}{3} \frac{\dot{V}}{V}-2 \frac{X_{1}}{V} .
$$

Now inserting (4.19) into (4.16), (4.17), (4.18) we finally find

$$
\begin{gathered}
\sigma_{1}^{1}=\sigma_{2}^{2}=\frac{X_{1}}{V}, \quad \sigma_{3}^{3}=-2 \frac{X_{1}}{V}, \\
\sigma^{2}=3 \frac{X_{1}^{2}}{V^{2}}, \\
A_{m}=18 \frac{X_{1}^{2}}{\dot{V}^{2}} .
\end{gathered}
$$

As it was shown in (4.8) and (4.9) in case of a positive $\lambda_{2}$ that generates late time acceleration, both $V$ and $\dot{V}$ becomes large with the expansion of the Universe leading to $\sigma_{i}^{i} \rightarrow 0, \sigma^{2} \rightarrow 0$ and $A_{m} \rightarrow 0$. This corresponds to our earlier conclusion regarding isotropization. As far as negative $\lambda_{2}$ is concerned, in this case the model gives rise to a oscillatory mode of expansion. In this case we have both local minima and maxima. The maximum (minimum) value of volume scale $V$ depends on the parameters and the initial condition and might be as large as possible. Hence in case of a negative $\lambda_{2}$ though it is possible to attain solution such that $\left.\sigma_{i}^{i}\right|_{V=V_{\max }} \rightarrow 0$ and $\left.\sigma^{2}\right|_{V=V_{\max }} \rightarrow 0$, 
Saha B.

but at the same time we have $\left.A_{m}\right|_{V=V_{\max }} \rightarrow \infty$ since at any spacetime point where $V=V_{\max (\min )}$ we have $\left.\dot{V}\right|_{V=V_{\max (\min )}}=0$. It means at any spacetime point where evolution changes its direction (expansion to contraction and vice versa) the Universe becomes highly anisotropic.

\section{CONCLUSIONS}

Within the scope of LRS Bianchi type-I cosmological model we have studied the role of spinor field in the evolution of the Universe. The reason for considering the LRS BI model lies on the fact that in case of a full Bianchi type-I model the non-diagonal components of the energymomentum tensor of the spinor fields imposes severe restrictions on the components of the spinor field resulting in vanishing scalar $S=\bar{\psi} \psi$ and pseudoscalar $P=\imath \bar{\psi} \gamma^{5} \psi[21,22]$. As a results both the mass tern and nonlinear tern in the Lagrangian disappears. But as it was shown here, in case of an LRS Bianchi type-I cosmological model neither mass term nor the nonlinear term vanish. Moreover, unlike Bianchi type-VI model the present model leads asymptotic isotropization. It is was also found that depending on the sign of self-coupling constant the model allows either accelerated mode of expansion or oscillatory mode of evolution.

\section{Acknowledgments}

This work is supported in part by a joint Romanian-LIT, JINR, Dubna Research Project, theme no. 05-6-1119-2014/2016.

[1] M Henneaux Phys. Rev. D21 857 (1980)

[2] U Ochs and M Sorg Int. J. Theor. Phys. 321531 (1993)

[3] B Saha and G N Shikin Gen. Relat. Grav. 291099 (1997)

[4] B Saha and G N Shikin J Math. Phys. 385305 (1997)

[5] B Saha Phys. Rev. D64 123501 (2001)

[6] C Armendáriz-Picón and P B Greene Gen. Relat. Grav. 351637 (2003)

[7] B Saha and T Boyadjiev Phys. Rev. D69 124010 (2004)

[8] B Saha Phys. Rev. D69 124006 (2004)

[9] M O Ribas, F P Devecchi and G M Kremer Phys. Rev. D72 123502 (2005)

[10] B Saha Phys. Particle. Nuclei. 37. Suppl. 1 S13 (2006) 
[11] B Saha Grav. \& Cosmol. 12(2-3)(46-47) 215 (2006)

[12] B Saha Romanian Rep. Phys. 59649 (2007)

[13] B Saha Phys. Rev. D74 124030 (2006)

[14] R C de Souza and G M Kremer Class. Quantum Grav. 25225006 (2008)

[15] G M Kremer and R C de Souza arXiv:1301.5163v1 [gr-qc] (2013)

[16] V G Krechet, M L Fel'chenkov and G N Shikin Grav. \& Cosmol. 14 No 3(55) 292 (2008)

[17] B Saha Cent. Euro. J. Phys. 8920 (2010)

[18] B Saha Romanian Rep. Phys. 62209 (2010)

[19] B Saha Astrophys. Space Sci. 331243 (2011)

[20] B Saha Int. J. Theor. Phys. 511812 (2012)

[21] B Saha Int. J. Theor. Phys. 531109 (2014)

[22] B Saha Astrophys. Space Sci. 35728 (2015)

[23] B Saha arXiv: 1504.03883V1 [gr-qc] (2015)

[24] B Saha arXiv: 1507.03847V1 [gr-qc] (2015)

[25] T W B Kibble J. Math. Phys. 2212 (1961)

[26] K A Bronnikov, E N Chudaeva and G N Shikin Class. Quantum Grav. 213389 (2004) 